\title{
COMMENTARY ON \\ "A BRIEF HISTORY OF THE CLASSIFICATION OF THE FINITE SIMPLE GROUPS" BY RONALD SOLOMON
}

\author{
ROBERT M. GURALNICK
}

ABSTRACT. Immediately following the commentary below, the previously published article is reprinted in its entirety: Ronald Solomon, "A brief history of the classification of the finite simple groups", Bull. Amer. Math. Soc. (N.S) 38 (2001), no. 3, 315-352.

The classification of finite simple groups is one of the momentous results in the history of mathematics. It has had an enormous impact on our understanding of finite groups. Many problems in finite group theory can be reduced to questions about the simple groups and their automorphisms, subgroup structure, and representations.

There have been enormous consequences of the classification in group and number theory, algebraic and arithmetic geometry, logic, and other areas. See the afterword (following the reprint of his article) by Ron Solomon for a very small sampling. See also the recent book of Smith 7] and the ICM article [5].

One of my favorite consequences is the fact that any finite transitive permutation group of degree $n>1$ contains an element of prime power order that has no fixed points (if one drops the prime power order condition, this is a very elementary theorem of Jordan). What is surprising about this result is that is equivalent to a fundamental result in number theory.

The proof of this theorem is also unique in the history of mathematics. As Solomon points out, the start of the proof is likely marked as in 1892 (in a remark by Hölder [6]) and it was not completed until 2004 with the publication of the two volumes of Aschbacher and Smith [2, 3. The proof is thousands of pages long, involved many researchers, and is likely the longest and most complicated proof of a theorem in mathematics.

One should also note that the theorem is not just a list. The theorem says that a typical finite simple group is basically a group of Lie type; i.e., it comes from a simple algebraic group (which corresponds to the simple Lie groups). This allows one to use tools from algebraic geometry and algebraic group theory to study the finite simple groups.

There are ongoing efforts to give second- and third-generation proofs of this as well. The first of these was initiated by Danny Gorenstein and Richard Lyons. The effort is now being led by Richard Lyons and Ron Solomon in a series of volumes

Received by the editors July 2, 2018.

2010 Mathematics Subject Classification. 20 D05.

The author was partially supported by the NSF grant DMS-1600056. 
published by the American Mathematical Society. So far seven volumes (and the Aschbacher-Smith books, which are an integral part of this new proof) have been published and, perhaps, another five are planned. See the very nice recent article by Solomon 8 giving a progress report on this effort. In this proof, one assumes the classification for all smaller groups, and this allows one to use properties of finite groups which depend on knowing the classification for all proper subgroups. This should simplify some of the proofs.

More recently, there have been efforts to abstract the "local" properties of finite groups to a more abstract setting: fusion systems. In this case, one works in a new category of finite $p$-groups and subgroups and morphisms between them (the model for this is a Sylow $p$-subgroup and its subgroups with the morphisms coming from conjugation in the group). Parts of the classification can be redone in this setting. The advantage of working in this category is that many tools from algebraic topology can be applied; see the article by Aschbacher and Oliver [1.

This beautifully written and fascinating article gives one some sense of the beauty and complications of the proof of the classification. As the title suggests, it is a brief history. There are necessarily many topics which are left out or only briefly discussed. For example, not too much is said about the discovery of the 26 sporadic groups, or the work of Tits, or the fundamental impact of the ideas of Thompson's $N$-group paper 9]. There is also a fascinating story of how Bombieri [4] finally proved that groups of Ree type are actually Ree groups (twisted versions in characteristic 3 of $G_{2}$ ).

\section{REFERENCES}

[1] Michael Aschbacher and Bob Oliver, Fusion systems, Bull. Amer. Math. Soc. (N.S.) 53 (2016), no. 4, 555-615, DOI 10.1090/bull/1538. MR.3544261

[2] Michael Aschbacher and Stephen D. Smith, The classification of quasithin groups. I: Structure of strongly quasithin K-groups, Mathematical Surveys and Monographs, vol. 111, American Mathematical Society, Providence, RI, 2004. MR2097623

[3] Michael Aschbacher and Stephen D. Smith, The classification of quasithin groups. II: Main theorems: the classification of simple QTKE-groups, Mathematical Surveys and Monographs, vol. 112, American Mathematical Society, Providence, RI, 2004. MR2097624

[4] Enrico Bombieri, A. Odlyzko, and D. Hunt, Thompson's problem $\left(\sigma^{2}=3\right)$, Invent. Math. 58 (1980), no. 1, 77-100, DOI 10.1007/BF01402275. Appendices by A. Odlyzko and D. Hunt. MR.570875

[5] Robert Guralnick, Applications of the classification of finite simple groups, Proceedings of the International Congress of Mathematicians-Seoul 2014. Vol. II, Kyung Moon Sa, Seoul, 2014, pp. 163-177. MR3728610

[6] Otto Hölder, Die einfachen Gruppen im ersten und zweiten Hundert der Ordnungszahlen (German), Math. Ann. 40 (1892), no. 1, 55-88, DOI 10.1007/BF01443490. MR.1510711

[7] Stephen D. Smith, Applying the classification of finite simple groups: A user's guide, Mathematical Surveys and Monographs, vol. 230, American Mathematical Society, Providence, RI, 2018. MR.3753581

[8] R. Solomon, The classification of finite simple groups: a progress report, Notices Amer. Math. Soc. 65 (2018), no. 6, 646-651.

[9] John G. Thompson, Nonsolvable finite groups all of whose local subgroups are solvable, Bull. Amer. Math. Soc. 74 (1968), 383-437, DOI 10.1090/S0002-9904-1968-11953-6. MR0230809

Department of Mathematics, University of Southern California, los Angeles, CalIFORNIA 90089-2532

Email address: guralnic@usc.edu 